\title{
Quantitative Autofluorescence Imaging of A375 Human Melanoma Cell Samples: A Pilot Study
}

\author{
Afshan Shirkavand ${ }^{1}$, Ezeddin Mohajerani ${ }^{2 *}$, Shirin Farivar ${ }^{3}$, Leila Ataie-Fashtami ${ }^{4}$, Mohammad Hossein \\ Ghazimoradi $^{5}$
}

'Laser and Plasma Research Institute, Shahid Beheshti University, Tehran, Iran; Department of Medical Laser, Medical Laser Research Center, Yara Institute, ACECR, Tehran, Iran

${ }^{2}$ Laser and Plasma Research Institute, Shahid Beheshti University, Tehran, Iran

${ }^{3}$ Department of Cell and Molecular Biology, Faculty of Life Sciences and Biotechnology, Shahid Beheshti University, Tehran, Iran

${ }^{4}$ Department of Regenerative Medicine, Royan Institute for Stem Cell Biology \& Technology, ACECR, Tehran, Iran ${ }^{5}$ Department of Cell and Molecular Biology, Faculty of Life Sciences and Biotechnology, Shahid Beheshti University, Tehran, Iran

\author{
*Correspondence to \\ Ezeddin Mohajerani, \\ Prof. in Photonics, Laser and \\ Plasma Research Institute, \\ Shahid Beheshti University \\ Tehran, Iran. \\ Tel: +98 9121990359, \\ Email: e-mohajerani@sbu.ac.ir
}

Published online February 14, 2021

\begin{abstract}
Introduction: Skin cancer is one of the most common types of malignancy worldwide. Human skin naturally contains several endogenous fluorophores, as potential sources can emit inherent fluorescence, called intrinsic autofluorescence (AF). The melanin endogenous fluorophore in the basal cell layer of the epidermis seems to have a strong autofluorescence signal among other ones in the skin. This pilot study aimed to investigate the feasibility of the detection of autofluorescence signals in the A375 human melanoma cell line in the cell culture stage using the FluoVision optical imaging system.

Methods: The human skin melanoma cell line (A375) donated as a gift from Switzerland (University Hospital Basel) was cultured. For the imaging of the A375 human melanoma cell sample in this pilot study, the FluoVision optical imaging device (Tajhiz Afarinan Noori Parseh Co) was applied. The proposed clustering image processing code was developed based on the K-mean segmentation method, using MATLAB software (version 16).

Results: The quantification of color pixels in the color bar along with the intensity score of the autofluorescence signal ranged between 0 and 70 was written in the image processing code execution and a threshold higher than $40 \%$, proportional to the ratio of autofluorescent cells. The percentage of the signal of A375 autofluorescent melanoma cells in the 3 studied cell samples was calculated as $3.11 \% \pm 0.6$.

Conclusion: This imaging method has the advantage of no need for fluorophore labels over the existing fluorescence imaging methods, and it can be regarded as one of the important choices of label-free imaging for this A375 melanoma cell line containing the intrinsic endogenous fluorophore in cell studies.

Keywords: Human Melanoma A375, Autofluorescence imaging, Segmentation, Quantitative, Image Processing
\end{abstract}

\section{Introduction}

Skin cancer is one of the most common types of malignancy worldwide. In recent decades, the prevalence of melanoma and non-melanoma skin cancers has been increasing. ${ }^{1-3}$ Malignant melanoma cancer is an aggressive skin cancer with a high recorded mortality rate, which is caused by the uncontrolled growth of melanocyte cells in the epidermis. ${ }^{4,5}$ Patients with advanced melanoma tend to have lower survival rates. Even though malignant melanoma accounts for about $1 \%$ of all skin cancers, most of the mortality reports relate to it. ${ }^{6,7}$ Human skin naturally contains several types of endogenous fluorophores that can potentially emit inherent fluorescence, autofluorescence without the need for labeling with exogenous fluorophores. These skin fluorophores include collagen, melanin, keratin, porphyrin, flavin, and $\operatorname{NAD}(\mathrm{P}) \mathrm{H} \cdot{ }^{8-10}$ Melanin is found in hair or basal cell layer of the epidermis, and has the strongest fluorescence signal among other skin tissue fluorophores. ${ }^{11}$ The imaging of these endogenous fluorophore molecules can be performed in a variety of imaging modes, among which optical imaging methods are very popular among 
researchers due to their being non-ionizing. Accordingly, attempts have been made to develop high-resolution optical techniques to detect the pathology of the studied tissues and to differentiate cancerous cells from noncancerous ones. ${ }^{12-14}$ Optical techniques used in in vitro and in vivo studies are optical microscopy, laser-induced fluorescence as well as fluorescence, diffuse reflectance spectroscopy as non-invasive, and non-ionizing imaging techniques. ${ }^{2,15,16}$ Concerning melanoma cancer cells which originate in melanocyte cells and contain a melanin endogenous fluorophore, the wavelength of the ultraviolet excitation light or near-infrared light can lead to the emission of fluorescence radiation from these molecules with recorded radiation at wavelengths of 440,520 , and $575 \mathrm{~nm} .{ }^{9,17}$ This optical excitation of fluorophores for fluorescence radiation can be performed by various optical laser sources or light-emitting diodes through selecting a wavelength appropriate for the optical absorption window related to that fluorophore. ${ }^{18,19}$ Excitation with low-power light in cells or tissue samples is non-destructive, providing a record of useful information on the biophysical and biochemical status of the sample. ${ }^{19,20}$

Since it is worthwhile to access non-destructive labelfree imaging methods for data acquisition in the in vitro studies, this pilot study aimed to investigate the feasibility of detecting the autofluorescence signal in the A375 human melanoma cell line using the FluoVision optical imaging system which has been introduced as a fluorescence imaging system in preclinical studies. We attempted to test the applicability of this system for the intrinsic fluorescence of melanoma cells. Furthermore, we aimed to develop a quantifying computer code for autofluorescence image processing for more accurate understanding.

\section{Materials and Methods Cell Culture}

To conduct this in vitro study, a sample of A375 human melanoma cell line was prepared as a gift from the cancer group in the Royan Research Institute. The A375 cell line was derived from a skin tissue sample of a 54-yearold woman with malignant melanoma. This cell line was used for culturing in Dulbecco's Modified Eagle Medium (DMEM) containing $1 \%$ glutamine, $1 \%$ penicillin and streptomycin (P/S), 1\% non-essential amino acids, and $10 \%$ fetal bovine serum (FBS) (all materials were purchased from Gibco). The cells were cultured at $37^{\circ} \mathrm{C}$ in the presence of $5 \% \mathrm{CO}_{2}$ in an incubator with $95 \%$ humidity. The cell culture medium was altered every 48 hours, and after about four days, when the cells reached appropriate confluency, they were separated from the bottom of the container by $0.05 \%$ trypsin and passaged, and then, while maintaining the culture conditions, they were propagated and cultured in triplicate in $3-\mathrm{cm}$ Petri dishes. ${ }^{3,21,22}$

\section{Autofluorescence Optical Imaging}

The FluoVision optical system (Tajhiz Afarinan Noori Parseh (TANP) Co, Tehran University of Medical Sciences) was selected for the imaging of the A375 human melanoma cell sample in this pilot study. ${ }^{13}$ The FluoVision imaging system is a non-invasive optical method utilizing non-ionizing light with the capability of being applied in preclinical studies. This imaging method can also be applied to detect cancer cells at the molecular level. In this method, the target area is marked with a fluorescent substance. The target area is then irradiated by the laser beam. The exciting area attempts to emit incoherent fluorescent radiations according to the Stokes shift. ${ }^{23}$ Fluorescent radiations are received by a very sensitive camera. In the fluorescent imaging method, the laser source and the detector are located on one side of the sample. This method of imaging is called optical imaging in a reflection mode where the fluorescent radiation is received by the CCD camera from the surface of the tissue as shown in Figure 1. The advantages of this imaging system include high sensitivity, flexibility in designing outdoor experiments, the ability to emit a single wavelength light or white light, and the ability to record data in real-time and on video. ${ }^{13}$

Due to the structure and characteristics of the FlouVision imaging system, and the endogenous melanin pigments in the melanoma cells, there was the probability of autofluorescence by appropriate excitation light. Autofluorescence imaging was performed with a light irradiation wavelength of $390 \mathrm{~nm}$ for the A375 cultured melanoma cell samples one day after cell passage. The experiment was done as a triple test.

\section{Quantification Algorithm}

Image segmentation is one of the most important methods of classifying information stored as image pixels and is regarded as one of the most important and appropriate methods used for decision-making applications in medical images as well. In the segmentation method, the image, based on its pixel information, is divided into several parts and separate regions, where pixels contain information sharing more similarities. There are several

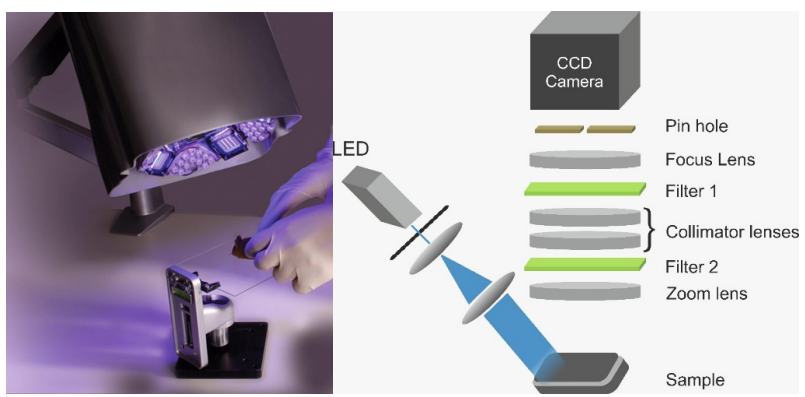

Figure 1. (a) FluoVision Imaging System and (b) its Optical Set-Up Schematic. ${ }^{13}$ 
techniques for image segmentation, including neural network and clustering methods. Among the clustering methods, different types of clustering are applicable, such as $\mathrm{K}$-means clustering and fuzzy C-means. The $\mathrm{K}$-means algorithm is one of the simplest and fastest image clustering methods that can be easily applied to process highly informative images.

To process the images obtained from the samples and to quantify the autofluorescence emission of the melanoma cell sample, coding with MATLAB software (version 16) was used. Our proposed clustering image processing code was developed based on the K-mean segmentation method, which is one of the clustering models. ${ }^{24,25}$ The flow chart of this image processing code is shown in Figure 2. In this clustering method, the image is divided into K-clusters based on similar pixel information in the neighborhood. In the first phase of processing, the center of the divided regions is calculated, and in the next phase, the closest distance from the center of one region to the next centers is determined. In this algorithm, Euclidean relations are applied to determine the closest distance of cluster centers to each other. ${ }^{26}$ Here, modeling of the basic $\mathrm{k}$-means algorithm is performed using Euclidian distance metric implementation.

In this section, we describe the algorithm flowchart shown in Figure 2 mathematically:

Let $\mathrm{X}=\{\mathrm{x} 1, \mathrm{x} 2, \mathrm{x} 3, \ldots \ldots, \mathrm{xn}\}$ be the set of data points and $\mathrm{C}=\{\mathrm{c} 1, \mathrm{c} 2, \ldots \ldots, \mathrm{cm}\}$ be the set of centers. The distance between each data point and cluster centers is calculated using the Euclidean distance metric as follows:

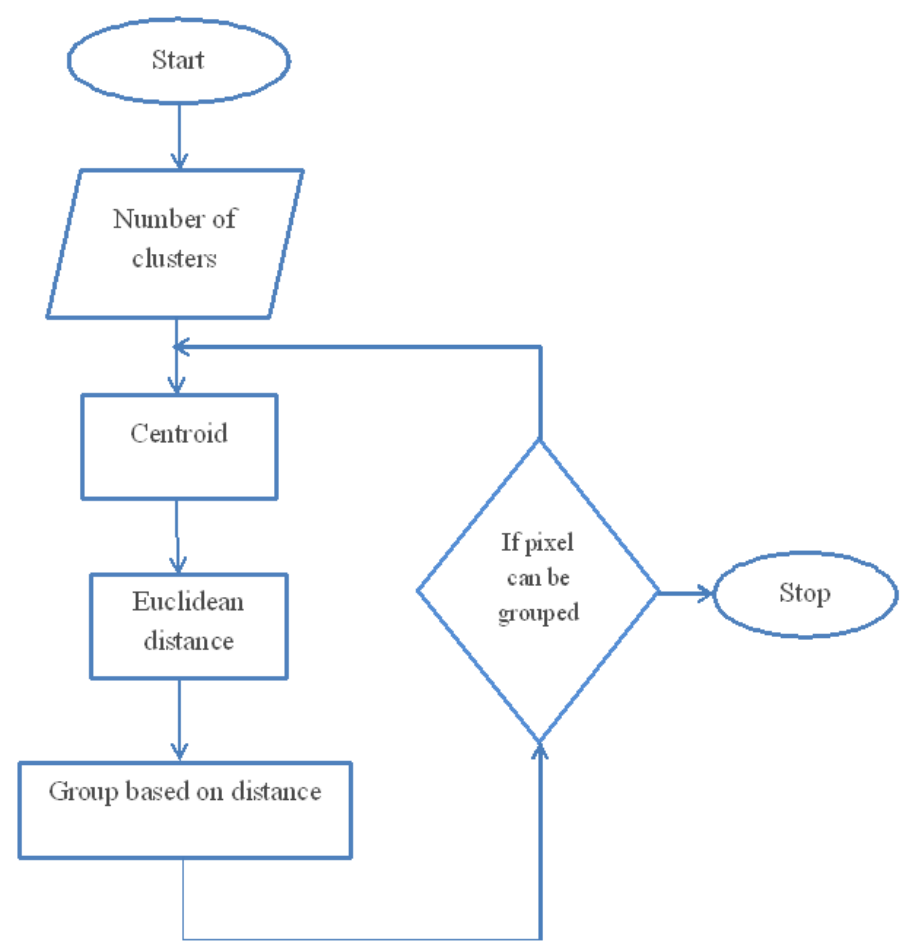

Figure 2. Flowchart of the Processor Executive Program Based on K-mean Segmentation in MATLAB Software. 


\section{melanoma cell image}

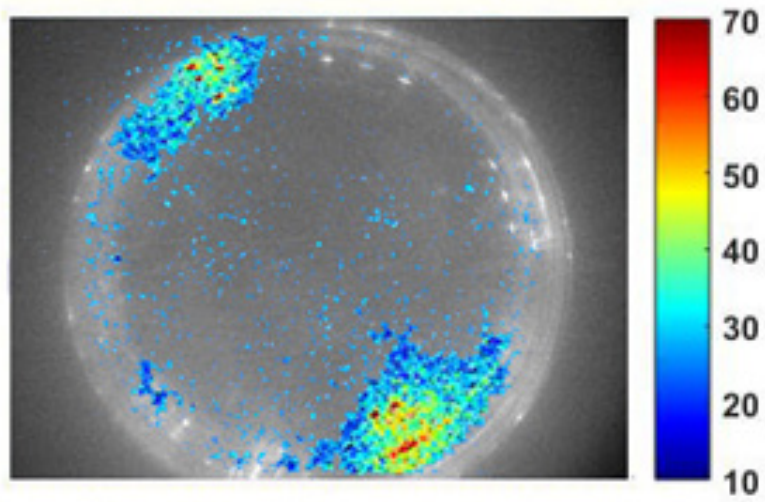

objects in cluster 3

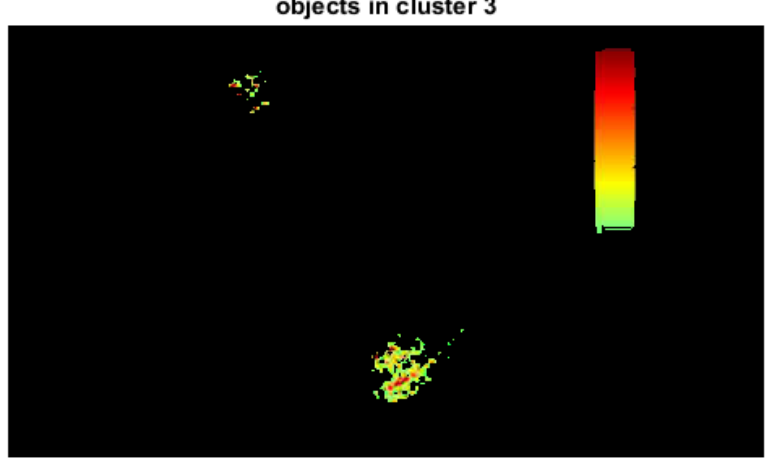

Figure 3. (a) A375 melanoma cell image fusion from raw data and excited with the autofluorescence wavelength (the color bar presents the percentage of the autofluorescent intensity), (b) the processed image depicting the threshold autofluorescence signal higher than $40 \%$.

system. As shown in this figure, the cancer cells have been proliferated on the sides of the culture plate, forming a mass. Referring to the emptier space in the image, there is a small fraction of cells emitting autofluorescence in our code quantifications as it is clear in Table 1.

\section{Discussion}

The autofluorescence of cells and tissues can provide extensive information about the cellular processes including changes in structure or function, health status, normal state, and being pathological or cancerous. As a label-free imaging technique, without the need for an exogenous fluorophore, just using available intrinsic fluorophores in the tissue or cell, autofluorescence is capable of investigating the alterations of biological samples, cellular function and detecting diseases in the early stages. ${ }^{10,27,28}$ Melanin clusters in melanin-containing cells like skin melanoma tissue can be investigated in terms of intrinsic fluorescence or autofluorescence radiation following the excitation of the intrinsic pigments by appropriate light wavelength irradiation.

This pilot study was conducted on autofluorescence obtained from melanoma cell samples containing a melanin intrinsic fluorophore in an in vitro laboratory model using the segmentation image processing to quantitatively analyze the autofluorescence signal of these cells. The set of image information obtained from the imaging of samples cultured in a cell culture dish can be entered into image processing software programs as input data and developed in the processor program. Moreover, these images are read as numerical matrices, and quantification analyses are carried out on them as well. Each pixel recorded in the image of the studied samples based on the recorded intensity of the resulting light can be regarded as a measure of the autofluorescence light of the melanoma cell sample. The initial result obtained from the imaging of these samples exhibited the potential of this system in the autofluorescence image of

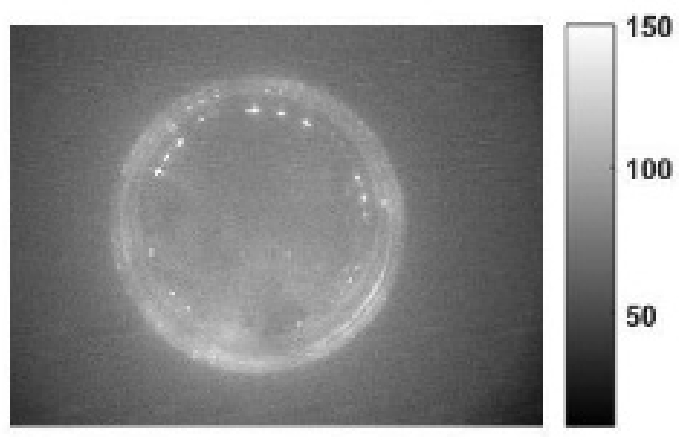

\section{Raw data}

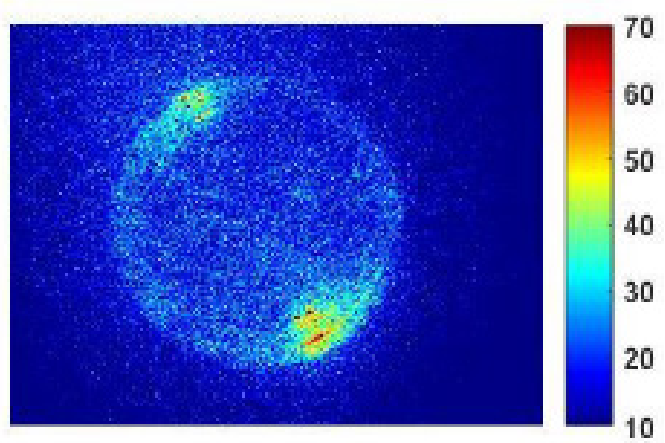

image with excited wavelength

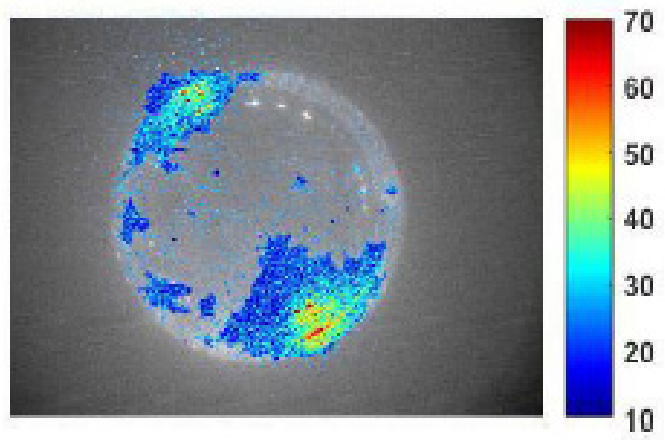

\section{image fusion}

Figure 4. The A375 Cell Culture Sample Image Obtained From the FluoVision Imaging System. 
Table 1. The Percentage of the Signal of A375 Autofluorescent Melanoma Cells in the Studied Cell Samples

\begin{tabular}{lcccc}
\hline Sample & $\mathbf{1}$ & $\mathbf{2}$ & $\mathbf{3}$ & Mean \pm SD \\
\hline Cell autofluorescent & $3.12 \%$ & $3.91 \%$ & $2.36 \%$ & $3.11 \% \pm 0.6$ \\
\hline
\end{tabular}

melanoma cells. Accordingly, this pilot study quantified the autofluorescence values of growing live cancer cells to investigate a limited number of A375 cultured melanoma cell samples and to develop a software processor program code using the MATLAB program. This imaging method has the advantage of no need for fluorophore labels over the existing fluorescence imaging methods, and it can be regarded as one of the important choices of label-free imaging for this A375 melanoma cell line containing an intrinsic endogenous fluorophore in cell studies with an intrinsic fluorophore.

Based on the initial results obtained from the images captured by the FluoVision system, the primary cultured cancer cells adhered to the sides of the plate and were growing. The empty space in the plate in the middle parts of the plate can be seen in Figure 4. Accordingly, it is recommended to carry out more comprehensive studies on various days after cell passage to investigate the filled status and the propagation of melanoma cancer cells as well as the impact of increasing cell confluency on quantitative changes in the recorded autofluorescence ratio.

The preliminary results of this study agree with the result of a study by Albertus et al who assessed the utility of autofluorescence determination in distinguishing melanoma and indeterminate nevomelanocytic lesions. They concluded that digital autofluorescence is capable of imaging the malignant lesions. ${ }^{29}$ Another study also showed that the autofluorescence approach allows the diagnosis of malignant melanomas with the accuracy for ex vivo and in vivo studies in line with our in vitro study. ${ }^{30}$

This imaging method and the developed image processing code, which result in the intensity of autofluorescence quantifications, seem to be feasible and promising for application as a quantified optical technique in cancerous and non-cancerous skin melanoma or treatment monitoring in melanoma studies. We have some more ongoing studies to investigate the treatment response monitoring of A375 melanoma cells and to evaluate the alterations of A375 melanoma cell autofluorescence on various days post-treatment. This might be applied in combination with other optical modalities such as reflectance spectroscopy.

As a pilot study, this research suffers from some limitations including the small number of cultured melanoma cell samples on the post-culture day. It would also be worthwhile to compare the cancerous and noncancerous cells; however, the normal skin cell lines like fibroblasts or adipose seem to be melanin-free and might lack melanin autofluorescence. Hence, more extensive ex vivo or in vivo studies are needed on normal and melanoma skin biopsy samples.

\section{Conclusion}

The quantification results of this study are promising, showing that this image processing technique is capable of quantitatively evaluating experimental studies in in vitro models. In addition, it seems that the technique has the potential for being applied in more extensive in vivo, ex vivo and preclinical studies. Depending on the presence of other endogenous chromophores in biological tissues, the method has the potential for being applied in various extensive studies.

\section{Ethical Considerations}

Human skin melanoma A375 cells were obtained from the Cancer department of Royan Institute not harvested for this research directly from human subject.

\section{Conflict of Interests}

There is no conflict of interest.

\section{Acknowledgment}

This study was conducted at Laser and Plasma Research Institute, Shahid Beheshti University. The authors would kindly like to thank Dr. Marzieh Ebrahimi from Cancer Group in the Royan Institute for her kind favor in providing us with human melanoma A375 (donated by Professor Spongli from the Oncology Center of the University Hospital of Basel, Switzerland).

\section{References}

1. Apalla Z, Lallas A, Sotiriou E, Lazaridou E, Ioannides D. Epidemiological trends in skin cancer. Dermatol Pract Concept. 2017;7(2):1-6. doi: 10.5826/dpc.0702a01.

2. Rajaram N, Aramil TJ, Lee K, Reichenberg JS, Nguyen TH, Tunnell JW. Design and validation of a clinical instrument for spectral diagnosis of cutaneous malignancy. Appl Opt. 2010;49(2):142-152. doi: 10.1364/AO.49.000142.

3. Shirkavand A, Farivar S, Mohajerani E, Ataie-Fashtami L, Ghazimoradi MH. Non-invasive reflectance spectroscopy for normal and cancerous skin cells refractive index determination: An in vitro study. Lasers Surg Med. 2019;51(8):742-50. doi: 10.1002/lsm.23095.

4. Cossu A, Casula M, Cesaraccio R, Lissia A, Colombino M, Sini MC, et al. Epidemiology and genetic susceptibility of malignant melanoma in North Sardinia, Italy. Eur J Cancer Prev. 2017;26(3):263-267. doi: 10.1097/ CEJ.0000000000000223.

5. Sini MC, Doneddu V, Paliogiannis P, Casula M, Colombino M, Manca A, et al. Genetic alterations in main candidate genes during melanoma progression. Oncotarget. 2018;9(9):8531-8541. doi: 10.18632/oncotarget.23989.

6. Siegel RL, Miller KD, Jemal A. Cancer statistics, 2019. CA Cancer J Clin. 2019;69(1):7-34. doi: 10.3322/caac.21551.

7. American Cancer Society. Cancer Facts \& Figures 2019. Atlanta: American Cancer Society; 2019. Available from: https://www.cancer.org/content/dam/cancer-org/research/ 
cancer-facts-and-statistics/annual-cancer-facts-andfigures/2019/cancer-facts-and-figures-2019.pdf.

8. Patalay R, Talbot C, Alexandrov Y, Munro I, Neil MA, König K, et al. Quantification of cellular autofluorescence of human skin using multiphoton tomography and fluorescence lifetime imaging in two spectral detection channels. Biomed Opt Express. 2011;2(12):3295-3308. doi: 10.1364/BOE.2.003295.

9. König K. Clinical multiphoton tomography. J Biophotonics. 2008;1(1):13-23. doi: 10.1002/jbio.200710022.

10. Koenig $\mathrm{K}$, Schneckenburger $\mathrm{H}$. Laser-induced autofluorescence for medical diagnosis. J Fluoresc. 1994;4(1):17-40. doi: 10.1007/BF01876650.

11. König K. Cellular response to laser radiation in fluorescence microscopes. In: Periasamy A, editor. Methods in cellular imaging. New York: Springer; 2001. p. 236-251. doi: 10.1007/978-1-4614-7513-2_14.

12. Miranda-Lorenzo I, Dorado J, Lonardo E, Alcala S, Serrano AG, Clausell-Tormos J, et al. Intracellular autofluorescence: A biomarker for epithelial cancer stem cells. Nat Methods. 2014;11(11):1161-1169. doi: 10.1038/nmeth.3112.

13. Ebrahimi SM, Seydi M, Ebrahimi M, Hejazi M. The Fluo Vision system for fluorescence concentration imaging. Front Biomed Technol. 2019;6(4):217-220. doi: 10.18502/ fbt.v6i4.2215.

14. Ra H, González-González E, Uddin MJ, King BL, Lee A, AliKhan I, et al. Detection of non-melanoma skin cancer by in vivo fluorescence imaging with fluorocoxib A. Neoplasia. 2015;17(2):201-207. doi: 10.1016/j.neo.2014.12.009.

15. Matthews TE, Piletic IR, Selim MA, Simpson MJ, Warren WS. Pump-probe imaging differentiates melanoma from melanocytic nevi. Sci Transl Med. 2011;3(71):71ra15. doi: 10.1126/scitranslmed.3001604

16. Zeng H, MacAulay C, Palcic B, Mclean DI. A computerized autofluorescence and diffuse reflectance spectroanalyser system for in vivo skin studies. Phys Med Biol. 1993;38(2):231-40. doi: 10.1088/0031-9155/38/2/002.

17. Sharikova AV. UV laser and LED induced fluorescence spectroscopy for detection of trace amounts of organics in drinking water and water sources [dissertations]. University of South Florida; 2009.

18. Lukinsone V, Kuzmina I, Veilande R, Plorina EV, Bliznuks $\mathrm{D}$, Bolochko K, et al. Multispectral and autofluorescence RGB imaging for skin cancer diagnostics. Proc. SPIE 11065, Saratov Fall Meeting 2018: Optical and Nano-Technologies for Biology and Medicine, 110650A (3 June 2019); https:// doi.org/10.1117/12.2518882.

19. Rodat-Boutonnet A, Naccache P, Morin A, Fabre J, Feurer B, Couderc F. A comparative study of LED-induced fluorescence and laser-induced fluorescence in SDS-CGE: Application to the analysis of antibodies. Electrophoresis. 2012;33(12):1709-1714. doi: 10.1002/elps.201200132.

20. Chernomyrdin NV, Lesnichaya AD, Yakovlev EV, Kudrin KG. Differentiation of basal cell carcinoma and healthy skin using multispectral modulation autofluorescence imaging : A pilot study. J Biomed Photonics Eng. 2019;5(1):010302. doi: 10.18287/JBPE19.05.010302.

21. Beazley-Long N, Gaston K, Harper SJ, Orlando A, Bates DO. Novel mechanisms of resistance to vemurafenib in melanoma - V600E B-Raf reversion and switching VEGF-A splice isoform expression. Am J Cancer Res. 2015;5(1):433441.

22. Dratkiewicz E, Simiczyjew A, Pietraszek-Gremplewicz K, Mazurkiewicz J, Nowak D. Characterization of melanoma cell lines resistant to vemurafenib and evaluation of their responsiveness to EGFR- and MET-inhibitor treatment. Int J Mol Sci. 2020;21(1):113. doi: 10.3390/ijms21010113.

23. Rovati L, Docchio F, Autofluorescence methods in ophthalmology. J Biomed Opt. 2004;9(1):9-21. doi: $10.1117 / 1.1628241$

24. Dhanachandra N, Manglem K, Chanu YJ. Image Segmentation Using K-means Clustering Algorithm and Subtractive Clustering Algorithm. Procedia Comput Sci. 2015;54:764-771. doi: 10.1016/j.procs.2015.06.090.

25. Gullanar MH, Nassir Hs. Image Segmentation Based on Image Histogram and Clustering Technique. Int J Graph Image Proc. 2015; 5(1):1-5

26. Singh A, Yadav A, Rana A. K-means with three different distance metrics. Int J Comput Appl. 2013;67(10):13-17.

27. Schneckenburger $\mathrm{H}$, Schmidt W. Luminescence techniques for monitoring of forest decline. J Photochem Photobiol B. 1992;13(2):190-193. doi: 10.1016/1011-1344(92)85058-3.

28. Tuite EM, Kelly JM. Photochemical interactions of methylene blue and analogues with DNA and other biological substrates. J Photochem Photobiol B Biol. 1993;21(2-3):103-124. doi: 10.1016/1011-1344(93)801737.

29. Albertus DL, Schachar IH, Zahid S, Elner VM, Demirci H, Jayasundera T. Autofluorescence quantification of benign and malignant choroidal nevomelanocytic tumors. JAMA Ophthalmol. 2013;131(8):1004-1008. doi: 10.1001/ jamaophthalmol.2013.4007.

30. Khristoforova YA, Bratchenko IA, Artemyev DN, Myakinin OO, Moryatov AA, Kozlov SV, et al. Method of autofluorescence diagnostics of skin neoplasms in the near infrared region. J Biomed Photonics Eng. 2016;1(3):186-92. doi: 10.18287/JBPE-2015-1-3-186. 